\title{
Comparison of hepatic and serum lipid signatures in hepatocellular carcinoma patients leads to the discovery of diagnostic and prognostic biomarkers
}

\author{
Yonghai Lu1,2,*, Juanjuan Chen ${ }^{3, *}$, Chong Huang ${ }^{4}$, Ning Li ${ }^{4}$, Li Zou ${ }^{1}$, Sin Eng Chia ${ }^{1}$, \\ Shengsen Chen ${ }^{4}$, Kangkang Yu ${ }^{4}$, Qingxia Ling ${ }^{4}$, Qi Cheng ${ }^{4}$, Mengqi Zhu ${ }^{4}$, Weidong \\ Zhang $^{5}$, Mingquan Chen ${ }^{4}$ and Choon Nam Ong ${ }^{1,6}$ \\ ${ }^{1}$ Saw Swee Hock School of Public Health, National University of Singapore, Singapore \\ ${ }^{2}$ Institute of Nutrition and Health, Qingdao University, Qingdao, Shandong, China \\ ${ }^{3}$ School of Marine Science, Ningbo University, Ningbo, Zhejiang, China \\ ${ }^{4}$ Department of Infectious Diseases and Hepatology of Huashan Hospital, Fudan University, Shanghai, China \\ ${ }^{5}$ School of Pharmacy, Second Military Medical University, Shanghai, China \\ ${ }^{6}$ NUS Environmental Research Institute, National University of Singapore, Singapore \\ *These authors contributed equally to this work \\ Correspondence to: Choon Nam Ong, email: ephocn@nus.edu.sg \\ Mingquan Chen, email: mingquanchen@fudan.edu.cn
}

Keywords: lipidomics, mass spectrometry, hepatocellular carcinoma, liver tissue, serum

Received: June 22, 2017 Accepted: September 03, 2017 Published: December 13, 2017

Copyright: Lu et al. This is an open-access article distributed under the terms of the Creative Commons Attribution License 3.0 (CC BY 3.0), which permits unrestricted use, distribution, and reproduction in any medium, provided the original author and source are credited.

\section{ABSTRACT}

We compared hepatic and serum lipid changes in hepatocellular carcinoma (HCC) patients to have a better understanding of the molecular pathogenesis of this disease and discovery novel lipid biomarkers. Hepatic and serum lipid profiling was conducted in paired liver and serum samples from 50 HCC patients and 24 healthy controls. A total of $\mathbf{2 0}$ hepatic and $\mathbf{4 0}$ serum lipid signatures were identified, yet there was hardly any significant correlation between them. The results indicated that triglycerides and phosphatidylcholines contributed significantly to altered hepatic lipids, whereas triglycerides and phosphatidylethanolamine-based plasmalogens (PEp) contributed most to altered serum lipids. In serum, PEp (36:4) and (40:6) showed a fair capability to discriminate HCC patients from healthy controls, and were significantly associated with HCC tumor grades $(p<0.05)$, and thus were identified as potential diagnostic and prognostic biomarkers of HCC. These findings were confirmed by a validation study conducted in an independent cohort consisting of 18 HCC, 20 cirrhosis patients, and $\mathbf{2 0}$ healthy controls. This study suggests that hepatic and serum lipid signatures of HCC have to be considered as mostly independent, and the results imply potential roles of PEp species, particularly PEp (36:4) and (40:6), as serum biomarkers for HCC diagnosis and progression.

\section{INTRODUCTION}

Hepatocellular carcinoma (HCC) is one of the most frequently encountering malignancies, marked by a poor 5 -year survival rate of less than $5 \%$. In addressing the demands for molecular diagnosis and therapy of HCC, extensive efforts have been made in the last two decades to identify altered expressed genes by genomics, proteins by proteomics, and metabolites by metabolomics in HCC patients [1-6]. In addition, it is worth noting that numerous lipid changes in $\mathrm{HCC}$ patients were reported in metabolomics studies [6-14].

Lipids are a group of naturally occurring hydrophobic molecules that play important roles in biological functions by storing energy, signaling, and acting as structural components of cell membranes [15]. 
Recent advances in lipidomics provided a new angle for lipid investigation in cancer research [16-22]. Yet to date, however, only a few lipidomics studies have been carried out to characterize hepatic [23-25] and serum $[26,27]$ lipid profiles of HCC patients. Previous studies showed the increase of phosphatidylcholines (PCs) [23] and the reduction of phosphatidylethanolamines (PEs), phosphatidylserines (PSs) and phosphatidylinositols (PIs) [25] in HCC tumors. In serum, most lipids were found to be diminished in HCC patients compared to healthy controls and hepatitis patients, including PCs, sphingomyelins (SMs), triglycerides (TGs), and cholesterol esters (CEs) [26]. Building on these findings, our current knowledge of the aberrant lipid metabolism in HCC is still in its infancy, leaving much of the fundamental aspects of their biology unknown. Additionally, the relationship between hepatic and serum lipid changes in HCC patients remains unclear. Liver plays a key role in lipid metabolism, including synthesis of lipoproteins and intracellular catabolism of lipids [28]. The aberrant lipid metabolism in liver may provide first-hand information on the development of liver diseases. Blood serum is a readily accessible and widely used homeostatic biofluid in clinic tests, providing information in regard to an individual's physiological status [29]. Therefore, better understanding of the correlation between hepatic and serum lipid changes could improve the molecular characterization of HCC and help to identify disease-relevant biomarkers.

In this study, we characterized both hepatic and serum lipid profiles of $50 \mathrm{HCC}$ patients using an untargeted lipidomics approach. When examining the lipid profiles, we aimed to compare the hepatic and serum lipid signatures in HCC patients, and also intended to identify the specific lipid biomarkers that could be used for diagnosis and progression of HCC.

\section{RESULTS}

\section{Demographic and clinical characteristics of subjects}

A total of 74 and 58 participants were respectively recruited from two study sites in this study, and their characteristics are shown in Table 1 and Supplementary Table 1. There were no significant differences on age and gender between groups in each batch. HCC patients had higher $\alpha$-fetoprotein (AFP) levels than liver cirrhosis patients $(p<0.05)$ (Supplementary Table 1$)$, and among HCC patients those who have cirrhosis also had higher AFP levels than those who do not have (Supplementary Table 2). Meanwhile, it was found that up to $40 \%$ (20 out of 50, Table 1) HCC patients had normal AF $P$ values (i.e., $<20 \mathrm{mg} / \mathrm{L}$ ) [30]. No significant differences were observed on alanine transaminase (ALT), aspartate transaminase (AST), and gamma-glutamyl transpeptidase (GGT) were found between $\mathrm{HCC}$ and liver cirrhosis patients
(Supplementary Table 1), and between HCC patients with and without liver cirrhosis (Supplementary Table 2).

\section{Hepatic and serum lipid signatures in HCC patients}

We started our investigation using a principal component analysis (PCA) model to explore the variances of lipid profiles among the three types of liver tissues. As illustrated in the score scatter plot (Supplementary Figure 1), hepatocellular carcinoma tissue (HCT) samples were separated from adjacent noncancerous tissue (ANT) and distal noncancerous tissue (DNT) samples with a few overlaps, while there was no significant separation between ANT and DNT samples. Besides, we compared the lipid profiles between two respective groups among these three types of liver tissues, respectively. All the results demonstrated that lipid profiles were not significantly different between ANT and DNT samples. Thus, we focused on the differences between HCT and DNT samples to investigate hepatic lipid signatures of HCC. As previous report [6], orthogonal partial leastsquares discriminant analysis (OPLS-DA) was applied to rank all lipids with respect to their performance for discriminating between HCT and DNT (Figure 1A). Finally, 20 lipid signatures were identified in HCC tumors, including six TGs, three PCs, three PEs, two phosphatidylglycerols (PGs), two PIs, two PSs, one SM, and one $\mathrm{CE}$ (Table 2). Their relative average quantities in HCT and DNT samples are shown in a heat map using $\mathrm{MeV}$ software (Supplementary Figure 2). Furthermore, OPLS-DA was applied to explore serum lipid signatures (Figure 1B). Finally, 40 lipid signatures were identified in serum, including 19 TGs, three lysophosphatidylcholines (LPCs), three PCs, eight PEs, one PI, three ceramides (Cers), one SM, and two CEs (Table 3). The relative average quantities of lipids in HCC patients and healthy controls are shown in Supplementary Figure 3. Stratification by cirrhosis status in HCC patients indicated that cirrhosis had no significant effects on the expression of most lipids in HCC patients (Supplementary Tables 3 and 4). On the basis of KEGG PATHWAY Database (http://www.genome.jp/kegg/) and literature, a map of HCC related lipid network was established based on these lipid signatures (Supplementary Figure 4).

\section{Comparison of hepatic and serum lipid signatures}

Among the hepatic and serum lipid signatures, only three lipids were common between them, they are PE (38:6), (40:6), and CE (22:6). Among them, PE (38:6) and (40:6) showed the same change trend in liver and serum, but CE (22:6) showed an opposite trend. Although TGs, PCs, PIs, SMs and CEs were changed in both liver and serum, these lipid species showed opposite trends. In addition, there was hardly any significant correlation between hepatic and serum lipid signatures in HCC 
Table 1: Characteristics of 50 hepatocellular carcinoma (HCC) patients and 24 healthy individuals

\begin{tabular}{llll}
\hline Characteristics ${ }^{\text {a }}$ & HCC patients & Healthy individuals & $\boldsymbol{P ~ v a l u e s ~}^{\mathbf{b}}$ \\
\hline No. of subjects & 50 & 24 & 0.766 \\
Gender (M/F) & $38 / 12$ & $19 / 5$ & 0.706 \\
Age (year) & $53(34 \sim 72)$ & $55(24 \sim 64)$ & \\
AFP (mg/L) & $45.3(1.62 \sim 24200)$ & - & \\
$\quad>20 /<20$ & $30 / 20$ & & \\
ALT (U/L) & $54(17 \sim 695)$ & - & \\
AST (U/L) & $63.5(20 \sim 903)$ & - & \\
GGT (U/L) & $86.5(11 \sim 647)$ & - & \\
HBsAg (positive/negative) & $46 / 4$ & $0 / 24$ & \\
HCVAb (positive/negative) & $1 / 49$ & $0 / 24$ & \\
Cirrhosis/no cirrhosis & $29 / 21$ & & \\
TNM stages & & & \\
T1N0M0 (early-stage) & 12 & & \\
T2N0M0 (early-stage) & 19 & & \\
T3N0M0 (late-stage) & 10 & & \\
T4N0M0 (late-stage) & 9 & & \\
\hline
\end{tabular}

a Age, AFP, ALT, AST, and GGT in HCC patients were expressed as median (range). Limitation on the number of subjects, T1 and T2 were set as early-stage of HCC; T3 and T4 were set as late-stage of HCC.

${ }^{\mathrm{b}} \mathrm{Chi}$-square test for gender, and Student's t-test for age.

patients (Supplementary Figure 5). These observations suggested that hepatic and serum lipid changes of HCC are rather difference and independent. With factor analysis (Table 4), it was found that TGs (factor 1) and PCs (factors 1 and 2) contributed most significantly towards the altered hepatic lipid metabolism in HCC patients, whereas TGs (factor 1) and phosphatidylethanolaminebased plasmalogens (PEp) (factor 2) contributed most significantly to the altered serum lipid metabolism.

\section{Diagnostic and prognostic capabilities of serum lipid signatures}

Receiver operating characteristic (ROC) analysis indicated that 24 out of 40 serum lipids showed a fair capability in diagnostic tests, with area under the curve (AUC) scores greater than 0.8 (Table 3). Of these, we found that PEp (36:4) and (40:6) showed a gradually decreased trend with the progression of HCC from earlyto late-stages (Figure 2A), and their levels were associated with TNM tumor stage $(p<0.05)$ (Supplementary Table 5). Although our data showed that HCC patients with cirrhosis had lower levels of PEp (36:4) and (40:6) than those without cirrhosis $(p<0.05)$ (Supplementary Table 4), the decreased trend of these two lipids from early- to late-stages were not affected by the cirrhosis status, as the frequency of HCC patients suffering from cirrhosis was similar in the two stage groups, $60 \%$ and $58 \%$ respectively. Furthermore, a classification with AUC equal to 0.880 was achieved when using the two lipids together to classify HCC patients and healthy subjects (Figure 2B). Our results tend to suggest that PEp (36:4) and (40:6) appear to be potential serum biomarkers for diagnosis and progression of HCC.

\section{External validation}

A semi-quantitative analysis of PEp (36:4) and (40:6) were carried out in the validation set to verify their capabilities for diagnosis and progression of HCC. Their levels were found to be significantly reduced in HCC and liver cirrhosis patients compared to healthy individuals, as well as their levels in HCC patients were much lower than those in liver cirrhosis patients (Figure 2C). Diagnostic tests showed that a combination of PEp (36:4) and (40:6) had good potential to discriminate HCC patients from both healthy individuals ( $\mathrm{AUC}=0.946)$ and liver cirrhosis patients $(A U C=0.732)$ (Figure 2D). Further, the c-statistic was increased from 0.732 in the basic model to 0.772 when putting AFP levels in the prediction model to differentiate $\mathrm{HCC}$ patients from liver cirrhosis patients (Supplementary Figure 6). The prediction probability values of the combined marker on HCC are shown in Figure 2E. At the cut-off value of 0.5 [31], 17 out of 18 HCC patients were correctly classified when compared to healthy individuals, giving a sensitivity of $89 \%$ and a specificity of $95 \%$; 13 were correctly classified when compared to liver cirrhosis patients, giving a sensitivity of $62 \%$ and a specificity of $71 \%$. It is worthy to note that the combination showed $80-90 \%$ diagnostic accuracy in 
Table 2: The 20 lipid signatures identified in the liver samples

\begin{tabular}{|c|c|c|c|c|c|c|c|c|}
\hline \multirow[t]{2}{*}{ Lipids } & \multirow[t]{2}{*}{ Candidates ${ }^{\text {a }}$} & \multirow{2}{*}{$\begin{array}{l}\text { R.T. } \\
\text { (min) }\end{array}$} & \multicolumn{3}{|c|}{$\mathbf{m} / \mathbf{z}$} & \multirow[t]{2}{*}{ Fragments } & \multirow[t]{2}{*}{ Formula } & \multirow[t]{2}{*}{ Trend $^{\mathrm{b}}$} \\
\hline & & & {$[\mathbf{M}+\mathbf{H}]^{+}$} & {$[\mathrm{M}+\mathrm{NH} 4]^{+}$} & {$[\mathbf{M}-\mathbf{H}]^{-}$} & & & \\
\hline \multicolumn{9}{|c|}{ Glycerolipids } \\
\hline $\mathrm{TG}(51: 0)$ & TG(16:0/17:0/18:0) [iso6] & 15.96 & & 866.8201 & & $593,579,565,267,253,239$ & C54H104O6 & Up \\
\hline $\mathrm{TG}(53: 1)$ & TG(16:0/18:1/19:0) [iso6] & 16.04 & & 892.8365 & & $619,593,577,281,265,239$ & C56H106O6 & Up \\
\hline $\mathrm{TG}(55: 2)$ & TG(15:0/18:1/22:1) [iso6] & 16.12 & & 918.8533 & & $645,619,563,321,265,225$ & C58H108O6 & Up \\
\hline $\mathrm{TG}(56: 1)$ & TG(16:0/18:1/22:0) [iso6] & 19.54 & & 934.8825 & & $661,635,577,323,265,239$ & C59H112O6 & Up \\
\hline $\mathrm{TG}(58: 1)$ & TG(16:0/18:1/24:0) [iso6] & 22.98 & & 962.9139 & & $689,663,577,351,265,239$ & C61H116O6 & Up \\
\hline $\mathrm{TG}(60: 2)$ & TG(16:0/18:1/26:1) [iso6] & 22.97 & & 988.9299 & & $715,689,577,377,265,239$ & C63H118O6 & Up \\
\hline \multicolumn{9}{|c|}{ Glycerophospholipids } \\
\hline $\mathrm{PC}(28: 0)$ & $\mathrm{PC}(14: 0 / 14: 0)$ & 6.69 & 678.5098 & & & $619,468,184$ & C36H72NO8P & Up \\
\hline $\mathrm{PC}(31: 0)$ & $\mathrm{PC}(15: 0 / 16: 0)$ [iso2] & 7.89 & 720.5581 & & & $661,494,480,184$ & C39H78NO8P & Up \\
\hline $\mathrm{PC}(35: 0)$ & $\operatorname{PC}(17: 0 / 18: 0)$ [iso2] & 9.27 & 776.6212 & & & $717,522,508,184$ & C43H86NO8P & $\mathrm{Up}$ \\
\hline $\operatorname{PE}(34: 2)$ & $\operatorname{PE}(16: 0 / 18: 2)$ [iso2] & 7.61 & & & 714.5101 & $279,255,196$ & C39H74NO8P & Down \\
\hline $\operatorname{PE}(38: 6)$ & $\operatorname{PE}(16: 0 / 22: 6)$ [iso2] & 7.38 & & & 762.5107 & $327,255,196$ & C43H74NO8P & Down \\
\hline $\operatorname{PE}(40: 6)$ & $\mathrm{PE}(18: 0 / 22: 6)$ [iso2] & 8.06 & & & 790.5404 & $327,283,196$ & C45H78NO8P & Down \\
\hline $\operatorname{PG}(36: 3)$ & $\operatorname{PG}(18: 1 / 18: 2)$ [iso2] & 6.51 & & & 771.5208 & $697,281,279,153$ & $\mathrm{C} 42 \mathrm{H} 77 \mathrm{O} 10 \mathrm{P}$ & Down \\
\hline $\operatorname{PG}(36: 4)$ & PG(18:2/18:2) & 5.99 & & & 769.5057 & $695,279,153$ & $\mathrm{C} 42 \mathrm{H} 75 \mathrm{O} 10 \mathrm{P}$ & Down \\
\hline $\operatorname{PI}(34: 2)$ & $\operatorname{PI}(16: 0 / 18: 2)$ [iso2] & 6.63 & & & 833.5204 & $577,553,279,255,153$ & C43H79O13P & Down \\
\hline $\operatorname{PI}(36: 2)$ & $\mathrm{PI}(18: 0 / 18: 2)$ [iso2] & 7.32 & & & 861.5523 & $581,577,283,279,153$ & $\mathrm{C} 45 \mathrm{H} 83 \mathrm{O} 13 \mathrm{P}$ & Down \\
\hline $\operatorname{PS}(38: 6)$ & $\operatorname{PS}(16: 0 / 22: 6)$ [iso2] & 6.83 & & & 806.4950 & $719,327,255,153$ & $\mathrm{C} 44 \mathrm{H} 74 \mathrm{NO} 10 \mathrm{P}$ & Down \\
\hline $\operatorname{PS}(40: 6)$ & $\operatorname{PS}(18: 0 / 22: 6)$ [iso2] & 7.47 & & & 834.5307 & $747,327,283,153$ & $\mathrm{C} 46 \mathrm{H} 78 \mathrm{NO} 10 \mathrm{P}$ & Down \\
\hline \multicolumn{9}{|c|}{ Sphingolipids } \\
\hline $\mathrm{SM}(\mathrm{d} 34: 2)$ & $\operatorname{SM}(\mathrm{d} 18: 1 / 16: 1)$ & 6.75 & 701.5615 & & & 683,184 & $\mathrm{C} 39 \mathrm{H} 77 \mathrm{~N} 2 \mathrm{O} 6 \mathrm{P}$ & Up \\
\hline \multicolumn{9}{|c|}{ Sterol lipids } \\
\hline $\mathrm{CE}(22: 6)$ & & 11.75 & & 714.6216 & & 369 & $\mathrm{C} 49 \mathrm{H} 76 \mathrm{O} 2$ & Up \\
\hline
\end{tabular}

${ }^{a}$ [iso6] means that the lipid might have six candidates/isomers, further analysis is needed for structure identification. Similar means for [iso2].

b "Up" and "Down" represent the lipid is up- and down-regulated in HCC tumor tissues compared to matched normal tissues, respectively.

these HCC patients with normal AF $P$ values $(<20 \mathrm{ng} / \mathrm{mL})$ (Figure 2F). Owing to the small samples size of HCC patients, we did not observer the significant association between PEp (36:4) and (40:6) levels with HCC tumor grade in the validation set (Supplementary Table 6), yet they still showed a gradually decreased trend (Supplementary Figure 7).

\section{DISCUSSION}

In order to reveal the relationship between hepatic and serum lipid changes in HCC, we first characterized both hepatic and serum lipid profiles of $50 \mathrm{HCC}$ patients and went further to identify the differential lipids. A total of 20 hepatic and 40 serum lipid signatures were identified in HCC patients, covering a wide range of lipid species including TGs, LPCs, PCs, PEs, PGs, PIs, PSs, Cers, SMs, and CEs. We noted that lipid metabolism in HCC tumors and sera are likely to be modulated in different manners, as most lipid species showed opposite tendency of changes in liver and serum, and only a few hepatic and serum lipids shared moderate correlations. Factor analysis confirmed that the altered hepatic lipid metabolism in HCC was mainly driven by TGs and PCs; the altered serum lipid metabolism was mainly driven by TG and PEp species.

Increased de novo lipogenesis is a common feature to most solid tumors, which occurs in tumourigenesis to supply energy and membrane lipids for accelerated cell proliferation [32]. TG is a precursor for synthesis of phospholipids, and also is a primary unit of energy storage [26], which is synthesized in liver beginning with acylation of glycerol-3-phosphate catalyzed by lysophosphatidic acid (LPA)-acyltransferase [33]. A recent study indicated that the isoleucine to methionine substitution at position 148 on patatin-like phospholipase domain-containing 3 (PNPLA3, adiponutrin) gene induced a gain of function in LPA-acyltransferase activity, leading to increased hepatic TG biosynthesis in HCC patients [34]. In addition, our previous study indicated that glycerol-3-phosphate significantly decreased in HCC tumors [6]. Taking them together, our current finding that TGs significantly increased in HCC tumors might be caused by the increased flux of glycerol-3-phosphate to TG in cancer cells. Besides de novo TG synthesis, HCC tumors also scavenge lipids from their environment. Previous study had indicated that uptake of fatty acids by cancer cells plays an important 
role in the development and progression of HCC [35]. Thus, enhanced TG uptake by HCC cancer cells from serum may also contribute to the increased TG levels in HCC tumors. It could also help explain why TG species reduced in serum of HCC patients as observed in our study and previous report [26], but further investigations are required to confirm these observations. Nevertheless, our study indicated an increase in TG synthesis/uptake in HCC tumors (Supplementary Figure 4).
Further, a series of glycerophospholipids were found to be altered significantly in HCC patients, including PCs, PEs, PGs, PIs, and PSs. Among them, PC and PE account for $40-60 \%$ of phospholipids of eukaryotic membranes and play important roles in cellular structure and biological functions [36]. A recent study showed that overexpression of LPC acyltransferase 1 enriched PC species in HCC tumors and promoted cell proliferation, which catalyzes the conversion of LPC to PC in the Lands cycle pathway of
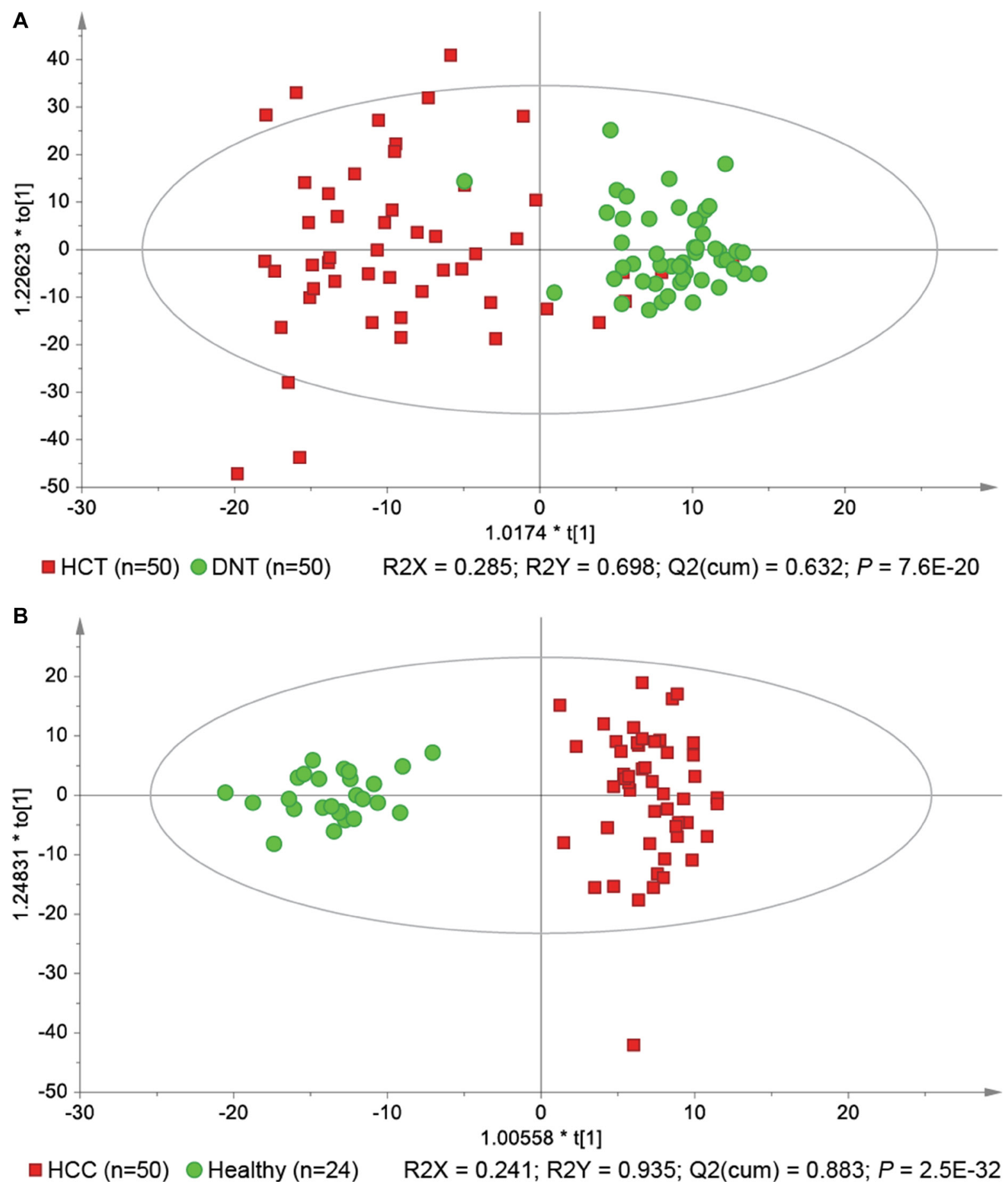

Figure 1: OPLS-DA score scatter plots show perturbations of hepatic (A) and serum (B) lipid profiles in hepatocellular carcinoma (HCC) patients. 
Table 3: The 40 lipid signatures identified in the serum samples

\begin{tabular}{|c|c|c|c|c|c|c|c|c|c|}
\hline \multirow[t]{2}{*}{ Lipids } & \multirow[t]{2}{*}{ Candidates ${ }^{\mathrm{a}}$} & \multirow{2}{*}{$\begin{array}{c}\text { R.T. } \\
(\min )\end{array}$} & \multicolumn{3}{|c|}{$\mathbf{m} / \mathbf{z}$} & \multirow[t]{2}{*}{ Fragments } & \multirow[t]{2}{*}{ Formula } & \multirow[t]{2}{*}{ Trend $^{b}$} & \multirow[t]{2}{*}{$\mathbf{A U C}$} \\
\hline & & & {$[\mathbf{M}+\mathbf{H}]+$} & {$[\mathrm{M}+\mathrm{NH} 4]+$} & [M-H]- & & & & \\
\hline \multicolumn{10}{|c|}{ Glycerolipids } \\
\hline $\mathrm{TG}(44: 1)$ & TG(12:0/14:0/18:1) [iso6] & 11.07 & & 766.6996 & & $549,521,467,265,211,183$ & C47H88O6 & Down & 0.76 \\
\hline $\mathrm{TG}(46: 0)$ & TG(12:0/16:0/18:0) [iso6] & 12.24 & & 796.7466 & & $579,523,495,267,239,183$ & С49H94O6 & Down & 0.80 \\
\hline TG(46:1) & TG(12:0/16:0/18:1) [iso6] & 11.59 & & 794.7312 & & $577,521,495,265,239,183$ & C49H92O6 & Down & 0.80 \\
\hline $\mathrm{TG}(46: 2)$ & TG(12:0/16:0/18:2) [iso6] & 11.12 & & 792.7155 & & $575,519,495,263,239,183$ & C49H90O6 & Down & 0.76 \\
\hline TG(46:3) & TG(12:0/16:0/18:3) [iso6] & 10.79 & & 790.6998 & & $573,517,495,261,239,183$ & C49H88O6 & Down & 0.65 \\
\hline $\mathrm{TG}(47: 0)$ & TG(15:0/16:0/16:0) [iso3] & 12.66 & & 810.7620 & & $551,537,239,225$ & $\mathrm{C} 50 \mathrm{H} 96 \mathrm{O} 6$ & Down & 0.75 \\
\hline $\mathrm{TG}(48: 0)$ & TG(16:0/16:0/16:0) & 13.10 & & 824.7780 & & 551,239 & C51H98O6 & Down & 0.88 \\
\hline $\mathrm{TG}(48: 1)$ & TG(14:0/16:0/18:1) [iso6] & 12.27 & & 822.7625 & & $577,549,523,265,239,211$ & C51H96O6 & Down & 0.81 \\
\hline $\mathrm{TG}(48: 2)$ & TG(14:0/16:0/18:2) [iso6] & 11.66 & & 820.7471 & & $575,547,523,263,239,211$ & C51H94O6 & Down & 0.77 \\
\hline TG(48:3) & TG(14:0/16:1/18:2) [iso6] & 11.36 & & 818.7314 & & $573,547,521,263,237,211$ & C51H92O6 & Down & 0.68 \\
\hline $\mathrm{TG}(49: 0)$ & TG(16:0/16:0/17:0) [iso3] & 13.67 & & 838.7937 & & $565,551,253,239$ & C52H100O6 & Down & 0.83 \\
\hline TG(49:1) & TG(16:0/16:1/17:0) [iso6] & 12.70 & & 836.7783 & & $565,563,549,253,239,237$ & C52H98O6 & Down & 0.84 \\
\hline $\mathrm{TG}(50: 0)$ & TG(16:0/16:0/18:0) [iso3] & 14.28 & & 852.8092 & & $579,551,267,239$ & C53H102O6 & Down & 0.94 \\
\hline $\mathrm{TG}(51: 1)$ & TG(16:0/17:0/18:1) [iso6] & 13.72 & & 864.8101 & & $591,577,565,265,253,239$ & C54H102O6 & Down & 0.85 \\
\hline $\mathrm{TG}(52: 0)$ & TG(16:0/18:0/18:0) [iso3] & 15.81 & & 880.8416 & & $607,579,267,239$ & C55H106O6 & Down & 0.94 \\
\hline $\mathrm{TG}(52: 1)$ & TG(16:0/18:0/18:1) [iso6] & 14.33 & & 878.8249 & & $605,579,577,267,265,239$ & C55H104O6 & Down & 0.93 \\
\hline $\mathrm{TG}(53: 1)$ & TG(17:0/18:0/18:1) [iso6] & 15.04 & & 892.8420 & & $605,593,591,267,265,253$ & C56H106O6 & Down & 0.87 \\
\hline $\mathrm{TG}(53: 2)$ & TG(17:0/18:1/18:1) [iso3] & 13.76 & & 890.8261 & & $603,591,265,253$ & С56H104O6 & Down & 0.86 \\
\hline $\mathrm{TG}(60: 10)$ & TG(18:0/20:4/22:6) [iso6] & 11.73 & & 972.8104 & & $671,651,627,311,287,267$ & C63H102O6 & Down & 0.87 \\
\hline \multicolumn{10}{|c|}{ Glycerophospholipids } \\
\hline LPC(18:3) & & 2.25 & 518.3275 & & & $500,258,184,104$ & $\mathrm{C} 26 \mathrm{H} 48 \mathrm{NO} 7 \mathrm{P}$ & Down & 0.77 \\
\hline LPC(20:5) & & 1.86 & 542.3276 & & & $524,258,184,104$ & $\mathrm{C} 28 \mathrm{H} 48 \mathrm{NO} 7 \mathrm{P}$ & Down & 0.72 \\
\hline LPC(22:6) & & 1.78 & 568.3458 & & & $550,258,184,104$ & $\mathrm{C} 30 \mathrm{H} 50 \mathrm{NO} 7 \mathrm{P}$ & Down & 0.86 \\
\hline $\mathrm{PC}(33: 0)$ & $\mathrm{PC}(15: 0 / 18: 0)$ [iso2] & 6.74 & 748.5811 & & & $689,522,480,184$ & $\mathrm{C} 41 \mathrm{H} 82 \mathrm{NO} 8 \mathrm{P}$ & Down & 0.80 \\
\hline $\mathrm{PC}(40: 7)$ & $\mathrm{PC}(18: 1 / 22: 6)$ [iso2] & 6.98 & 832.5880 & & & $773,566,520,184$ & C48H82NO8P & Down & 0.76 \\
\hline $\mathrm{PC}(40: 9)$ & PC(18:3/22:6) [iso2] & 7.13 & 828.5597 & & & $769,566,516,184$ & $\mathrm{C} 48 \mathrm{H} 78 \mathrm{NO} 8 \mathrm{P}$ & Down & 0.75 \\
\hline $\operatorname{PE}(36: 4)$ & PE(16:0/20:4) [iso2] & 7.36 & & & 738.5105 & $303,255,196$ & $\mathrm{C} 41 \mathrm{H} 74 \mathrm{NO} 8 \mathrm{P}$ & Down & 0.79 \\
\hline $\mathrm{PE}(38: 6)$ & PE(16:0/22:6) [iso2] & 7.24 & & & 762.5046 & $327,255,196$ & $\mathrm{C} 43 \mathrm{H} 74 \mathrm{NO} 8 \mathrm{P}$ & Down & 0.81 \\
\hline $\operatorname{PE}(40: 6)$ & $\mathrm{PE}(18: 0 / 22: 6)$ [iso2] & 7.90 & & & 790.5393 & $327,283,196$ & $\mathrm{C} 45 \mathrm{H} 78 \mathrm{NO} 8 \mathrm{P}$ & Down & 0.80 \\
\hline $\operatorname{PEp}(36: 4)$ & PE(P-16:0/20:4) & 7.67 & & & 722.5156 & $436,303,259,196$ & $\mathrm{C} 41 \mathrm{H} 74 \mathrm{NO} 7 \mathrm{P}$ & Down & 0.83 \\
\hline $\operatorname{PEp}(38: 4)$ & PE(P-18:0/20:4) & 8.33 & & & 750.5464 & $464,303,259,196$ & $\mathrm{C} 43 \mathrm{H} 78 \mathrm{NO} 7 \mathrm{P}$ & Down & 0.75 \\
\hline $\operatorname{PEp}(38: 6)$ & $\mathrm{PE}(\mathrm{P}-16: 0 / 22: 6)$ & 7.52 & & & 746.5135 & $436,327,283,196$ & $\mathrm{C} 43 \mathrm{H} 74 \mathrm{NO} 7 \mathrm{P}$ & Down & 0.87 \\
\hline $\operatorname{PEp}(40: 6)$ & PE(P-18:0/22:6) & 8.19 & & & 774.5468 & $464,327,283,196$ & $\mathrm{C} 45 \mathrm{H} 78 \mathrm{NO} 7 \mathrm{P}$ & Down & 0.82 \\
\hline $\operatorname{PEp}(40: 7)$ & PE(P-18:1/22:6) & 7.64 & & & 772.5317 & $462,327,283,196$ & $\mathrm{C} 45 \mathrm{H} 76 \mathrm{NO} 7 \mathrm{P}$ & Down & 0.81 \\
\hline $\operatorname{PI}(36: 4)$ & $\mathrm{PI}(18: 2 / 18: 2)$ & 4.69 & & & 857.5140 & $577,279,153$ & $\mathrm{C} 45 \mathrm{H} 79 \mathrm{O} 13 \mathrm{P}$ & Up & 0.78 \\
\hline \multicolumn{10}{|c|}{ Sphingolipids } \\
\hline $\operatorname{Cer}(\mathrm{d} 32: 0)$ & Cer(d18:0/14:0) & 8.04 & 512.5089 & & & $494,282,264,252$ & $\mathrm{C} 32 \mathrm{H} 65 \mathrm{NO} 3$ & Up & 0.88 \\
\hline Cer(d38:0) & Cer(d18:0/20:0) & 10.00 & 596.6013 & & & $578,282,264,252$ & $\mathrm{C} 38 \mathrm{H} 77 \mathrm{NO} 3$ & Up & 0.70 \\
\hline $\operatorname{Cer}(\mathrm{d} 40: 0)$ & Cer(d18:0/22:0) & 10.50 & 624.6342 & & & $606,282,264,252$ & $\mathrm{C} 40 \mathrm{H} 81 \mathrm{NO} 3$ & Up & 0.66 \\
\hline $\mathrm{SM}(\mathrm{d} 42: 1)$ & SM(d18:1/14:0) & 6.42 & 675.5510 & & & 657,184 & $\mathrm{C} 37 \mathrm{H} 75 \mathrm{~N} 2 \mathrm{O} 6 \mathrm{P}$ & Down & 0.89 \\
\hline \multicolumn{10}{|c|}{ Sterol lipids } \\
\hline $\mathrm{CE}(18: 1)$ & & 12.92 & & 668.6399 & & 369 & $\mathrm{C} 45 \mathrm{H} 78 \mathrm{O} 2$ & Down & 0.71 \\
\hline $\mathrm{CE}(22: 6)$ & & 11.44 & & 714.6257 & & 369 & $\mathrm{C} 49 \mathrm{H} 76 \mathrm{O} 2$ & Down & 0.86 \\
\hline
\end{tabular}

${ }^{a}$ [iso6] means that the lipid might have six candidates/isomers, further analysis is needed for structure identification. Similar means for [iso3] and [iso2].

" "Up" and "Down" represent the lipid is up- and down-regulated in serum of HCC patients compared to health individuals, respectively.

${ }^{\mathrm{c}} \mathrm{AUC}$ value of ROC analysis between HCC patients and healthy subjects. 

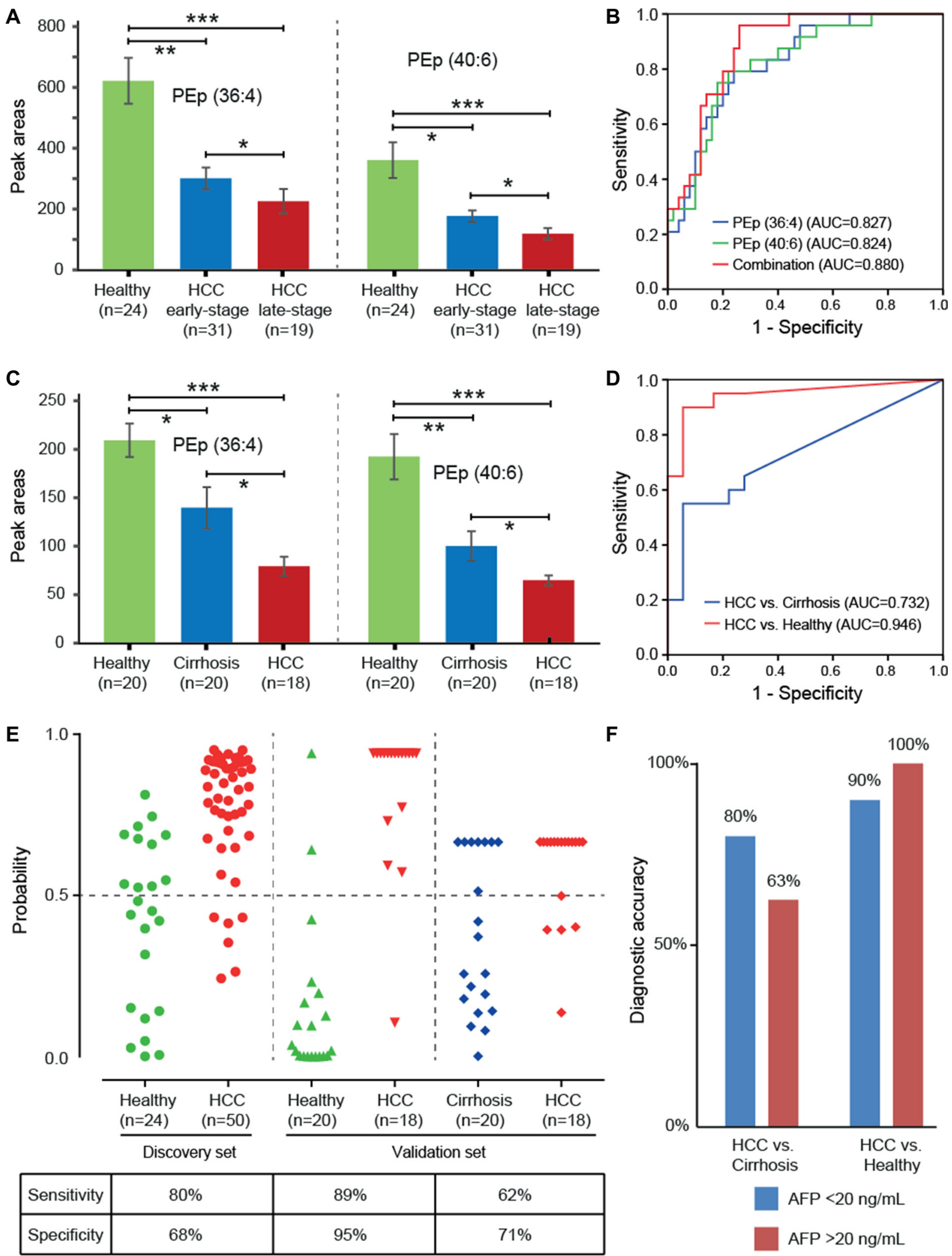

Figure 2: Diagnostic capabilities of PEp (36:4) and (40:6) for hepatocellular carcinoma (HCC). (A) Altered expressions of PEp (36:4) and (40:6) in serum of healthy subjects, early-stage HCC patients, and late-stage HCC patients; (B) ROC curve of serum PEp (36:4) and (40:6) in the discovery set; (C) Altered expressions of PEp (36:4) and (40:6) in serum of healthy subjects, liver cirrhosis patients, and HCC patients; (D) ROC curve of the combination of PEp (36:4) and (40:6) in the validation set; (E) Discrimination of healthy subjects, liver cirrhosis patients, and HCC patients by using the combined serum levels of PEp (36:4) and (40:6), at a cut-off of probability of 0.5 ; (F) Diagnostic accuracy of the combined marker PEp (36:4) and (40:6) for HCC patients with different concentrations of AFP in the validation set. 
Table 4: Percentage of variances and main loadings (loading value $>0.5$ ) explained by first two factors for hepatic and serum lipid changes in hepatocellular carcinoma (HCC) patients

\begin{tabular}{lcccc}
\hline \multirow{2}{*}{ \% of Variance } & \multicolumn{3}{c}{ Liver } & \multicolumn{2}{c}{ Serum } \\
\cline { 2 - 5 } Factor 1 (31.8\%) & Factor 2 (14.6\%) & Factor 1 (36.6\%) & Factor 2 (14.9\%) \\
\hline Main Loadings & $\mathrm{TG}(51: 0)$ & $\mathrm{PC}(31: 0)$ & $\mathrm{TG}(46: 3)$ & $\operatorname{PEp}(36: 4)$ \\
$\mathrm{TG}(53: 1)$ & $\mathrm{PE}(40: 6)$ & $\mathrm{TG}(48: 0)$ & $\operatorname{PEp}(38: 4)$ \\
$\mathrm{TG}(55: 2)$ & $\mathrm{PS}(40: 6)$ & $\mathrm{TG}(48: 1)$ & $\operatorname{PEp}(38: 6)$ \\
$\mathrm{TG}(56: 1)$ & & $\mathrm{TG}(48: 2)$ & $\mathrm{PEp}(40: 6)$ \\
$\mathrm{TG}(58: 1)$ & $\mathrm{TG}(48: 3)$ & $\mathrm{PEp}(40: 7)$ \\
$\mathrm{TG}(60: 2)$ & $\mathrm{TG}(49: 1)$ & $\mathrm{PC}(40: 7)$ \\
$\mathrm{PC}(28: 0)$ & $\mathrm{TG}(50: 0)$ & \\
$\mathrm{PC}(35: 0)$ & $\mathrm{TG}(51: 1)$ & \\
& & $\mathrm{TG}(52: 0)$ & \\
& & $\mathrm{TG}(52: 1)$ & \\
& & $\mathrm{TG}(53: 1)$ & $\mathrm{TG}(53: 2)$ \\
\hline
\end{tabular}

PC biosynthesis [24], which supports our finding that the elevated PC species in HCC tumors. In addition, we found that PC species were significantly decreased in serum of HCC patients, which was consistent with previous study as well [26]. In this study, five plasmalogens were found to be significantly reduced in serum of HCC patients compared to healthy controls, including PEp (36:4), $(38: 4),(38: 6),(40: 6)$ and $(40: 7)$. Plasmalogens are a type of ether phospholipids characterized by fatty alcohols with a vinyl ether linkage at sn-1 position and polyunsaturated fatty acids (PUFAs) with an ester linkage at sn-2 position. Although functions of plasmalogens have not yet been fully elucidated, it has been reported that they can reduce the damaging effects of reactive oxygen species in cancer cells [37]. To date, serum plasmalogen deficiencies have been linked to several cancers (e.g., colon cancer, prostate cancer, lung cancer, breast cancer, ovary cancer, and kidney cancer) [38].

Another important feature observed in HCC patients was the significant reduction of docosahexaenoic acid (DHA)-content glycerophospholipids, including LPC (22:6), PC (40:7), (40:9), PE (38:6), (40:6), PEp (38:6), (40:6), (40:7), and PS (38:6), (40:6) (Table 2 and 3). DHA is an omega-3 PUFA, which can be synthesized from alpha-linolenic acid, but mostly obtained directly from the diet. In 2009, Lim et al. found that omega-3 PUFAs such as DHA and eicosapentaenoic acid (EPA) could inhibit HCC cancer cell growth by blocking $\beta$-catenin and cyclooxygenase-2 [39]. The reduction of DHA-content glycerophospholipids observed in our study might be because of self-protection consumption, in which DHA was released from glycerophospholipids for self-care intervention. It is worth noting that our previous study also indicated a reduction in PUFAs (e.g., linoleic acid) in HCC tumors [6].
Ideally, the biomarkers used for disease diagnosis and progression should be easily accessible in clinical tests. Therefore, we only investigated the diagnostic and prognostic potential of serum lipid signatures for HCC. Although TGs, particularly TG (50:0), (52:0) and (52:1), showed the highest potential in the diagnosis of HCC with AUC $>0.9$, their changes were not associated with the progression of HCC. Conversely, we found that the changes of PEp (36:4) and (40:6) were significantly correlated with TNM tumor grade $(p<0.05)$, and they also showed a moderate potential for HCC diagnosis (AUC $>0.8$ ). In this study, we aimed to find the lipid biomarkers with both diagnostic and prognostic capabilities, and thus we focused on the changes of PEp (36:4) and (40:6) in the validation study. The results confirmed that the two lipids could differentiate HCC patients form liver cirrhosis patients and healthy subjects with high sensitivity and specificity (Figure 2E). More importantly, they showed high diagnostic accuracy for HCC patients with low AF $P$ values, providing a supplementary role to AFP for HCC diagnosis (Figure 2F).

The strengths of this study include the use of paired liver and serum samples and a well-established MS technique. This study is among the first to investigate the correlation between hepatic and serum lipid changes in HCC patients using an untargeted lipidomics method. Here, some limitations of our study still need to be acknowledged. First, the validation study was conducted within a limited number of participants. Further, in the validation phase, PEp (36:4) and (40:6) were only semiquantitative because of no available standards. Therefore, the two candidate biomarkers from this study should be validated in a large replication cohort using standards.

In summary, a wide range of hepatic and serum lipid signatures was identified in HCC patients, including 
TGs, LPCs, PCs, PEs, PGs, PIs, PSs, Cers, SMs, and CEs. Compared with the limited lipid studies of HCC, our results indicated that hepatic and serum lipid changes in $\mathrm{HCC}$ patients might be modulated in different manners. The overall data indicated that PEp (36:4) and (40:6) showed significant diagnostic potential for $\mathrm{HCC}$, and their levels were associated with TNM tumor grade. External validation verified that they are two candidate diagnostic biomarkers of $\mathrm{HCC}$, while their potential roles as prognostic biomarkers of $\mathrm{HCC}$ need to be investigated further.

\section{MATERIALS AND METHODS}

\section{Participants and sample collection}

A batch of $50 \mathrm{HCC}$ patients and 24 healthy volunteers were recruited at the Shanghai Huashan Hospital (Shanghai, China) between February 2011 and August 2012 (Table 1). Due to the limitation of subject numbers, 50 HCC patients were simply classified into early- and latestages according to TNM staging system for progression investigation of $\mathrm{HCC}$, in which $\mathrm{T} 1$ and T2 were set as early-stage of HCC $(n=31)$; T3 and T4 were set as latestage of HCC $(n=19)$. Of these, 46 HCC patients were hepatitis B surface antigen (HBsAg) positive, and one was anti-hepatitis $\mathrm{C}$ virus (HCV) positive. Hepatic lipid profiling of $50 \mathrm{HCC}$ patients was conducted by using a pairwise comparison of HCC tumor tissues and matched normal liver tissues of each patient. Three types of liver tissue were collected as surgical specimens from each HCC patient during surgery: HCT was from the central area of the solid tumor, ANT was collected at 1-2 cm surrounding the solid tumor, and DNT was collected at 5 $\mathrm{cm}$ away from the solid tumor. The tissue samples were snap-frozen with liquid nitrogen followed by storage at $-80^{\circ} \mathrm{C}$ until analysis. Serum lipid profiling of $50 \mathrm{HCC}$ patients was carried out by comparison with the 24 healthy subjects. The morning fasting antecubital venous blood samples were collected from the participants using a sterile 21 -gage needle syringe, allowed to clot at $4{ }^{\circ} \mathrm{C}$ overnight and centrifuged at $1,699 \mathrm{~g}$ for $10 \mathrm{~min}$ to obtain sera. The serum samples were stored in Eppendorf micro-tubes at $-80^{\circ} \mathrm{C}$ until analysis. These 150 liver tissue and 74 serum samples were used as a discovery set for the identification of potential lipid signatures. The lipid signatures with diagnostic and prognostic potential were further evaluated in an independent batch of 58 serum samples from 18 HCC patients, 20 liver cirrhosis patients, and 20 healthy individuals, as described in the Supplemental Materials and Methods. The 58 subjects were recruited at the Jurong People's Hospital (Jiangsu, China) between April 2013 and June 2014 (Supplementary Table 1). Among them, 15 $\mathrm{HCC}$ and 14 liver cirrhosis patients were HBsAg positive, and $3 \mathrm{HCC}$ and 6 liver cirrhosis patients were anti-HCV positive. All participants voluntarily joined this study, gave written informed consent, and completed a questionnaire that provided demographical information including age, gender, lifestyle factors, and medical family history. The study protocols were approved by the Institutional Review Boards at the Huashan Hospital, Jurong People's Hospital and National University of Singapore, and conducted in accordance with the Helsinki Declaration of 1975, as revised in 1996.

\section{Sample preparation}

Tissue extraction was performed using a modified method to that described by Roberts et al. [40]. Frozen liver tissue $(5 \pm 0.05 \mathrm{mg})$ was mixed with $400 \mu \mathrm{L}$ of chloroform/methanol (2:1, v/v) containing $2.5 \mu \mathrm{g} / \mathrm{mL}$ PC (15:0) and $5 \mu \mathrm{g} / \mathrm{mL}$ PC (17:0/17:0) as internal standards. The mixture was homogenized using a TissueLyser LT (Qiagen, UK) at $25 \mathrm{~Hz}$ for $10 \mathrm{~min}$, then sonicated for 15 minutes. Following this, $200 \mu \mathrm{L}$ of chloroform and $200 \mu \mathrm{L}$ of water were added, then centrifuged for 10 minutes at $20,187 \mathrm{~g}$. The organic phase was collected and filtered by Thermo Scientific ${ }^{\mathrm{TM}}$ national $750 \mu \mathrm{L}$ microcentrifugal filters (PTFE membrane, $0.2 \mu \mathrm{m}$ pore size, non-sterile). The filtrate was dried under nitrogen gas, then reconstituted in $200 \mu \mathrm{L}$ chloroform/methanol $(2: 1, \mathrm{v} / \mathrm{v})$ for LC-MS analysis. Serum lipids were extracted using Bligh \& Dyer's method [41] with minor modifications. Serum $(100 \mu \mathrm{L})$ was diluted with $900 \mu \mathrm{L}$ of chloroform/methanol/ water $(2: 1: 1, \mathrm{v} / \mathrm{v} / \mathrm{v})$ containing $2.5 \mu \mathrm{g} / \mathrm{mL}$ PC (15:0) and 5 $\mu \mathrm{g} / \mathrm{mL}$ PC (17:0/17:0) as internal standards. The mixture was shaken vigorously for $5 \mathrm{~min}$, and then centrifuged for 20 minutes at 20,187 g. The organic phase was collected and dried under nitrogen gas. The dry residues were reconstituted in $200 \mu \mathrm{L}$ chloroform/methanol $(2: 1, \mathrm{v} / \mathrm{v})$ for LC-MS analysis. Quality control (QC) samples were separately prepared for tissue and serum samples, and analyzed to evaluate the stability and reproducibility of LC-MS analytical system (Supplementary Figure 8).

\section{LC-MS analysis}

Lipid profiling was performed on an Agilent 1290 ultrahigh pressure LC system (Waldbronn, Germany) coupled to a 6540 Q-ToF mass spectrometer equipped with an electrospray ionization (ESI) source. The samples were analyzed in both ESI-positive and -negative ion modes. The separation was performed on a phenomenex Kinetex C18 column $(50 \times 2.1 \mathrm{~mm}, 2.6 \mu \mathrm{m})$ at $40^{\circ} \mathrm{C}$. The mobile phases were methanol/acetonitrile/isopropyl alcohol $(9: 4: 2, \mathrm{v} / \mathrm{v} / \mathrm{v})$ with $20 \mathrm{mM} \mathrm{HCOONH}_{4}$ (A) and water/ isopropyl alcohol $(13: 2, \mathrm{v} / \mathrm{v})$ with $20 \mathrm{mM} \mathrm{HCOONH}_{4}$ (B). The gradient program was: $0-5 \mathrm{~min}, 60-85 \% \mathrm{~A} ; 5-10 \mathrm{~min}$, $85-100 \%$ A; $10-25 \mathrm{~min}, 100 \% \mathrm{~A} ; 25-26 \mathrm{~min}, 100-60 \%$ A. The flow rate was set at $0.4 \mathrm{~mL} / \mathrm{min}$. A $10 \mu \mathrm{L}$ of sample was loaded for each individual analysis. Mass data were acquired between $\mathrm{m} / \mathrm{z} 100$ and 1200 at a rate of two scans per second. The ion spray voltage was set at $4,000 \mathrm{~V}$, and 
the heated capillary temperature was maintained at $350^{\circ} \mathrm{C}$. The drying gas and nebulizer nitrogen gas flow rates were 12.0 L/min and $50 \mathrm{psi}$, respectively. MS/MS analysis was carried out for the identification and characterization of lipid candidates with multiple collision energies, including 10, 20 and $40 \mathrm{~V}$. Lipid identifications were conducted by searching the MS/MS data against entries in the Lipid MAPS (http://www.lipidmaps.org/) and METLIN (http:// metlin.scripps.edu/) databases with mass errors of $<5 \mathrm{ppm}$. We named lipid species as combined the number of carbons and the number of double bonds, as well as the candidate isomers. A workflow of manual identification of lipids used in this study is shown in Supplementary Figure 9.

\section{Data pre-processing and statistical analysis}

The hepatic and serum spectral data were exported as mzData files, and respectively pretreated by opensource software MZmine version 2.8 for peak detection, peak alignment and peak area normalization, in which the data of each sample was normalized to total area to correct for the MS response shift from the first injection to the last injection. The preprocessed lipid data were screened by using " $80 \%$ rule" and missing values (i.e., zeros) were replaced by $1 / 2$ minimum $[42,43]$ before statistical analysis. Analytical reliability of LC-MS method was assessed by a PCA model. The differential hepatic lipids in HCC patients were screened by comparing HCT and DNT samples. The lipids with variable importance in the projection (VIP) values of $>1.0$ in the orthogonal partial least squares discriminant analysis (OPLS-DA) model and $p$ values of $<0.05$ in the paired t test were considered as signatures. Serum lipid signatures in HCC patients were defined by VI $P$ values of $>1.0$ in the OPLS-DA model and $p$ values of $<0.05$ in the Student's t test when compared to healthy subjects. Pearson correlation analysis were performed to reveal the significant correlation between hepatic and serum lipid changes. Factor analysis was applied to reduce dimensions of lipidomics data. ROC analysis was used to evaluate the diagnostic potential of lipids. SIMCA-P 14.0 software was used for multivariate statistical analyses (PCA and OPLS-DA). The paired $t$ test, Student's t test, Pearson correlation analysis, factor analysis and ROC analysis were performed in IBM SPSS Statistics 24 software. $P<0.05$ was considered significant. The false discovery rate method of Benjamini and Yekutieli was used to correct for multiple hypothesis testing \& reduce false positives.

\section{Abbreviations}

HCC: hepatocellular carcinoma; PCs: phosphatidylcholines; PEs: phosphatidylethanolamines; PSs: phosphatidylserines; PIs: phosphatidylinositols; SMs: sphingomyelins, TGs: triglycerides; CEs: cholesteryl esters; AFP: alpha-fetoprotein; ALT: alanine transaminase; AST: aspartate transaminase; GGT: gammaglutamyl transpeptidase; PCA: principal component analysis; HCT: hepatocellular carcinoma tissue; ANT: adjacent noncancerous tissue; DNT: distal noncancerous tissue; OPLS-DA: orthogonal partial least-squares discriminant analysis; PGs: phosphatidylglycerols; LPCs: lysophosphatidylcholines; Cers: ceramides; PEp: phosphatidylethanolamine-based plasmalogens; ROC: receiver operating characteristic; AUC: area under the curve; LPA: lysophosphatidic acid; PNPLA3: patatin-like phospholipase domain-containing 3; PUFAs: polyunsaturated fatty acids; DHA: docosahexaenoic acid; EPA: eicosapentaenoic acid; HBsAg: hepatitis B surface antigen; HCV: hepatitis C virus; QC: quality control; ESI: electrospray ionization; VIP: variable importance in the projection.

\section{ACKNOWLEDGMENTS AND FUNDING}

This study was partially supported by the grant of Natural Science Fund of The Science and Technology Commission of Shanghai, China (12ZR1404300), Science and Technology Commission of Shanghai Municipality (16411954300), NUS secondment Funds to CNO (706000-005-133) and NUS Environmental Research Institute (NERI) (608-000-007-731). The funder had no role in study design, data collection and analysis, decision to publish, or preparation of the manuscript.

\section{CONFLICTS OF INTEREST}

The authors have no conflicts of interest to declare.

\section{REFERENCES}

1. Okabe H, Satoh S, Kato T, Kitahara O, Yanagawa R, Yamaoka Y, Tsunoda T, Furukawa Y, Nakamura Y. Genomewide analysis of gene expression in human hepatocellular carcinomas using cDNA microarray: identification of genes involved in viral carcinogenesis and tumor progression. Cancer Res. 2001; 61:2129-2137.

2. Tran PL, Vigneron JP, Pericat D, Dubois S, Cazals D, Hervy M, DeClerck YA, Degott C, Auclair C. Gene therapy for hepatocellular carcinoma using non-viral vectors composed of bis guanidinium-tren-cholesterol and plasmids encoding the tissue inhibitors of metalloproteinases TIMP-2 and TIMP-3. Cancer Gene Ther. 2003; 10:435-444.

3. El-Aneed A, Banoub J. Proteomics in the diagnosis of hepatocellular carcinoma: focus on high risk hepatitis B and C patients. Anticancer Res. 2006; 26:3293-3300.

4. Lee NP, Cheung ST, Poon RT, Fan ST, Luk JM. Genomic and proteomic biomarkers for diagnosis and prognosis of hepatocellular carcinoma. Biomark Med. 2007; 1:273-284.

5. Khattab MA, Eslam M, Mousa YI, Ela-adawy N, Fathy S, Shatat M, Abd-Aalhalim H, Kamal A, Sharawe MA. Association between metabolic abnormalities and hepatitis 
C-related hepatocellular carcinoma. Ann Hepatol. 2012; 11:487-494.

6. Lu Y, Li N, Gao L, Xu YJ, Huang C, Yu K, Ling Q, Cheng Q, Chen S, Zhu M, Fang J, Chen M, Ong CN. Acetylcarnitine is a candidate diagnostic and prognostic biomarker of hepatocellular carcinoma. Cancer Res. 2016; 76:2912-2920.

7. Gao H, Lu Q, Liu X, Cong H, Zhao L, Wang H, Lin D. Application of $1 \mathrm{H}$ NMR-based metabonomics in the study of metabolic profiling of human hepatocellular carcinoma and liver cirrhosis. Cancer Sci. 2009; 100:782-785.

8. Patterson AD, Maurhofer O, Beyoglu D, Lanz C, Krausz KW, Pabst T, Gonzalez FJ, Dufour JF, Idle JR. Aberrant lipid metabolism in hepatocellular carcinoma revealed by plasma metabolomics and lipid profiling. Cancer Res. 2011; 71:6590-6600.

9. Zhou L, Wang Q, Yin P, Xing W, Wu Z, Chen S, Lu X, Zhang Y, Lin X, Xu G. Serum metabolomics reveals the deregulation of fatty acids metabolism in hepatocellular carcinoma and chronic liver diseases. Anal Bioanal Chem. 2012; 403:203-213.

10. Beyoglu D, Imbeaud S, Maurhofer O, Bioulac-Sage P, Zucman-Rossi J, Dufour JF, Idle JR. Tissue metabolomics of hepatocellular carcinoma: tumor energy metabolism and the role of transcriptomic classification. Hepatology. 2013; 58:229-238.

11. Huang Q, Tan Y, Yin P, Ye G, Gao P, Lu X, Wang H, Xu G. Metabolic characterization of hepatocellular carcinoma using nontargeted tissue metabolomics. Cancer Res. 2013; 73:4992-5002.

12. Liu Y, Hong Z, Tan G, Dong X, Yang G, Zhao L, Chen X, Zhu Z, Lou Z, Qian B, Zhang G, Chai Y. NMR and LC/MSbased global metabolomics to identify serum biomarkers differentiating hepatocellular carcinoma from liver cirrhosis. Int J Cancer. 2014; 135:658-668.

13. Fitian AI, Nelson DR, Liu C, Xu Y, Ararat M, Cabrera R. Integrated metabolomic profiling of hepatocellular carcinoma in hepatitis $\mathrm{C}$ cirrhosis through GC/MS and UPLC/MS-MS. Liver Int. 2014; 34:1428-1444.

14. Lu Y, Huang C, Gao L, Xu YJ, Chia SE, Chen S, Li N, Yu K, Ling Q, Cheng Q. Identification of serum biomarkers associated with hepatitis B virus-related hepatocellular carcinoma and liver cirrhosis using mass-spectrometrybased metabolomics. Metabolomics. 2015; 11:1526-1538.

15. Subramaniam S, Fahy E, Gupta S, Sud M, Byrnes RW, Cotter D, Dinasarapu AR, Maurya MR. Bioinformatics and systems biology of the lipidome. Chem Rev. 2011; 111:6452-6490.

16. Li J, Ren S, Piao HL, Wang F, Yin P, Xu C, Lu X, Ye G, Shao Y, Yan M, Zhao X, Sun Y, Xu G. Integration of lipidomics and transcriptomics unravels aberrant lipid metabolism and defines cholesteryl oleate as potential biomarker of prostate cancer. Sci Rep. 2016; 6:20984.

17. Buas MF, Gu H, Djukovic D, Zhu J, Drescher CW, Urban N, Raftery D, Li CI. Identification of novel candidate plasma metabolite biomarkers for distinguishing serous ovarian carcinoma and benign serous ovarian tumors. Gynecol Oncol. 2016; 140:138-144.

18. Zhang Y, Liu Y, Li L, Wei J, Xiong S, Zhao Z. High resolution mass spectrometry coupled with multivariate data analysis revealing plasma lipidomic alteration in ovarian cancer in Asian women. Talanta. 2016; 150:88-96.

19. Hilvo M, Denkert C, Lehtinen L, Muller B, Brockmoller S, Seppanen-Laakso T, Budczies J, Bucher E, Yetukuri L, Castillo S, Berg E, Nygren H, Sysi-Aho M, et al. Novel theranostic opportunities offered by characterization of altered membrane lipid metabolism in breast cancer progression. Cancer Res. 2011; 71:3236-3245.

20. Marien E, Meister M, Muley T, Fieuws S, Bordel S, Derua R, Spraggins J, Van de Plas R, Dehairs J, Wouters J, Bagadi M, Dienemann H, Thomas M, et al. Non-small cell lung cancer is characterized by dramatic changes in phospholipid profiles. Int J Cancer. 2015; 137:1539-1548.

21. Ishikawa S, Tateya I, Hayasaka T, Masaki N, Takizawa Y, Ohno S, Kojima T, Kitani Y, Kitamura M, Hirano S, Setou M, Ito J. Increased expression of phosphatidylcholine (16:0/18:1) and (16:0/18:2) in thyroid papillary cancer. PloS One. 2012; 7:e48873.

22. Zhao YY, Cheng XL, Lin RC. Lipidomics applications for discovering biomarkers of diseases in clinical chemistry. Int Rev Cell Mol Biol. . 2014; 313:1-26.

23. Abel S, De Kock M, van Schalkwyk DJ, Swanevelder S, Kew MC, Gelderblom WC. Altered lipid profile, oxidative status and hepatitis B virus interactions in human hepatocellular carcinoma. Prostaglandins Leukot Essent Fatty Acids. 2009; 81:391-399.

24. Morita Y, Sakaguchi T, Ikegami K, Goto-Inoue N, Hayasaka T, Hang VT, Tanaka H, Harada T, Shibasaki Y, Suzuki A, Fukumoto K, Inaba K, Murakami M, et al. Lysophosphatidylcholine acyltransferase 1 altered phospholipid composition and regulated hepatoma progression. J Hepatol. 2013; 59:292-299.

25. Krautbauer S, Meier EM, Rein-Fischboeck L, Pohl R, Weiss TS, Sigruener A, Aslanidis C, Liebisch G, Buechler C. Ceramide and polyunsaturated phospholipids are strongly reduced in human hepatocellular carcinoma. Biochim Biophys Acta. 2016; 1861:1767-1774.

26. Chen S, Yin P, Zhao X, Xing W, Hu C, Zhou L, Xu G. Serum lipid profiling of patients with chronic hepatitis B, cirrhosis, and hepatocellular carcinoma by ultra fast LC/ITTOF MS. Electrophoresis. 2013; 34:2848-2856.

27. Li J, Hu C, Zhao X, Dai W, Chen S, Lu X, Xu G. Largescaled human serum sphingolipid profiling by using reversed-phase liquid chromatography coupled with dynamic multiple reaction monitoring of mass spectrometry: method development and application in hepatocellular carcinoma. J Chromatogr A. 2013; 1320:103-110.

28. Jiang JT, Xu N, Zhang XY, Wu CP. Lipids changes in liver cancer. J Zhejiang Univ Sci B. 2007; 8:398-409.

29. Bird SS, Marur VR, Sniatynski MJ, Greenberg HK, Kristal BS. Serum lipidomics profiling using LC-MS and high- 
energy collisional dissociation fragmentation: focus on triglyceride detection and characterization. Anal Chem. 2011; 83:6648-6657.

30. Shen QJ, Fan J, Yang XR, Tan YX, Zhao WF, Xu Y, Wang N, Niu YD, Wu Z, Zhou J, Qiu SJ, Shi YH, Yu B, et al. Serum DKK1 as a protein biomarker for the diagnosis of hepatocellular carcinoma: a large-scale, multicentre study. Lancet Oncol. 2012; 13:817-826.

31. Zeng J, Yin PY, Tan YX, Dong LW, Hu CX, Huang Q, Lu X, Wang HY, Xu GW. Metabolomics study of hepatocellular carcinoma: discovery and validation of serum potential biomarkers by using capillary electrophoresis-mass spectrometry. J Proteome Res. 2014; 13:3420-3431.

32. Zhang F, Du G. Dysregulated lipid metabolism in cancer. World J Biol Chem. 2012; 3:167-174.

33. Kawano Y, Cohen DE. Mechanisms of hepatic triglyceride accumulation in non-alcoholic fatty liver disease. J Gastroenterol Hepatol. 2013; 48:434-441.

34. Kumari M, Schoiswohl G, Chitraju C, Paar M, Cornaciu I, Rangrez AY, Wongsiriroj N, Nagy HM, Ivanova PT, Scott SA, Knittelfelder O, Rechberger GN, BirnerGruenberger R, et al. Adiponutrin functions as a nutritionally regulated lysophosphatidic acid acyltransferase. Cell Metab. 2012; 15:691-702.

35. Cao D, Song X, Che L, Li X, Pilo MG, Vidili G, Porcu A, Solinas A, Cigliano A, Pes GM, Ribback S, Dombrowski F, Chen $\mathrm{X}$, et al. Both de novo synthetized and exogenous fatty acids support the growth of hepatocellular carcinoma cells. Liver Int. 2016.
36. Zhou X, Lawrence TJ, He Z, Pound CR, Mao J, Bigler SA. The expression level of lysophosphatidylcholine acyltransferase 1 (LPCAT1) correlates to the progression of prostate cancer. Exp Mol Pathol. 2012; 92:105-110.

37. Nagan N, Zoeller RA. Plasmalogens: biosynthesis and functions. Prog Lipid Res. 2001; 40:199-229.

38. Mankidy R, Ahiahonu PW, Ma H, Jayasinghe D, Ritchie SA, Khan MA, Su-Myat KK, Wood PL, Goodenowe DB. Membrane plasmalogen composition and cellular cholesterol regulation: a structure activity study. Lipids Health Dis. 2010; 9:62.

39. Lim K, Han C, Dai Y, Shen M, Wu T. Omega-3 polyunsaturated fatty acids inhibit hepatocellular carcinoma cell growth through blocking beta-catenin and cyclooxygenase-2. Mol Cancer Ther. 2009; 8:3046-3055.

40. Roberts LD, West JA, Vidal-Puig A, Griffin JL. Methods for performing lipidomics in white adipose tissue. Methods Enzymol. 2014; 538:211-231.

41. Bligh EG, Dyer WJ. A rapid method of total lipid extraction and purification. Can J Biochem Phys. 1959; 37:911-917.

42. Bijlsma S, Bobeldijk I, Verheij ER, Ramaker R, Kochhar S, Macdonald IA, van Ommen B, Smilde AK. Large-scale human metabolomics studies: a strategy for data (pre-) processing and validation. Anal Chem. 2006; 78:567-574.

43. Smilde AK, van der Werf MJ, Bijlsma S, van der Werff-van der Vat BJ, Jellema RH. Fusion of mass spectrometry-based metabolomics data. Anal Chem. 2005; 77:6729-6736. 